\title{
Accurate L-Band Measurements of the Dielectric Constant of Seawater
}

\author{
R. H. Lang*, C. Utku*, J. Janiczek*, Y. Tarkocin*, D. M. Le Vine** \\ *Dept. of Electrical \& Computer Engineering, George Washington University \\ Washington, DC 20052 USA - email: lang@gwu.edu \\ **Instrumentation Sciences Branch, Code 614.6, NASA/GSFC \\ Greenbelt, MD 20771 USA
}

\begin{abstract}
A new temperature controlled microwave cavity system to measure the complex dielectric constant of seawater at $1.413 \mathrm{GHz}$ is discussed. The system is being developed to measure seawater for temperatures from $0^{\circ} \mathrm{C}$ to $30^{\circ} \mathrm{C}$ and salinities from 10 to 40 psu, The paper discusses the construction of the measurement system and initial stability tests.
\end{abstract}

\section{Keywords-seawater; salinity; dielectric constant; microwave}

\section{INTRODUCTION}

This paper describes a resonant cavity technique for the measurement of the dielectric constant of seawater as a function of its salinity and temperature at $1.413 \mathrm{GHz}$. Accurate relationships between salinity/temperature and dielectric constant of the seawater are needed for sensor systems such as SMOS and Aquarius that will monitor salinity from space in the near future. The purpose of these new measurements is to establish the dependence of the dielectric constant of seawater on salinity and temperature more accurately than measurements performed in the past, to firmly establish error bounds for the measurements and to make the measurements in modern psu units.

The original parameterization of the seawater dielectric constant as a function of frequency, salinity and temperature by Klein and Swift [1] is based in part on measurements made by Ho and Hall [2] at $2.650 \mathrm{GHz}$ and $\mathrm{Ho}$, et al [3] at $1.430 \mathrm{GHz}$ (slightly removed from 1.413). Ho and coauthors measured the complex dielectric constant of seawater by introducing a small amount of seawater into a resonant cavity and then measuring the frequency shift of the resonance peak and the change in the $\mathrm{Q}$ factor of the cavity. The measurements were performed in the early 1970 's using a reflection cavity technique. This paper describes a new seawater experiment using the transmission resonant cavity technique which has greater accuracy and less heating of the sample. More recently, preliminary measurements using the cavity technique have been given by Lang et al [4]. Blanch and Aguasca [5] who employed a strip line approach have also reported measurement results.

An outline of the paper's organization is as follows: In section II, the basic technique for making the measurements is described. Following this in section III, construction of the cavity and preliminary measurements are discussed. In section IV, the new temperature control system is described, and in section $\mathrm{V}$, the measurement procedure is discussed along with the results of some initial stability tests. The paper concludes with a short summary in section VI.

\section{Resonant CAVIty Technique}

The resonant cavity technique has been used in the past for making highly accurate dielectric measurements. The technique involves the use of a high $Q$ cylindrical cavity of known resonance frequency $f_{o}$, and quality factor $Q_{o}$, which operates using a $\mathrm{TE}_{011}$ or a $\mathrm{TM}_{010}$ mode. Specifically, the cavity used in this experiment is designed to operate at the $\mathrm{TM}_{010}$ mode since the $\mathrm{TE}_{011}$ mode is degenerate. A liquid sample is introduced into the cavity via a slender glass tube with a hole in the center. The dielectric constant of the liquid perturbs the fields inside the cavity causing a change in both the resonance frequency and the cavity $Q$. If the perturbation is small, the changes in the resonance frequency and the $Q$ of the cavity are related to the real and imaginary parts of the liquid dielectric constant respectively. As a result, the dielectric constant of the liquid can be retrieved by the following perturbation relations:

$$
\begin{aligned}
& \varepsilon_{r}^{\prime}-1=2 C \Delta f / f_{o} \quad, \quad \Delta f=f_{o}-f \\
& \varepsilon_{r}^{\prime \prime}=C \Delta(1 / Q) \quad, \quad \Delta(1 / Q)=1 / Q-1 / Q_{o}
\end{aligned}
$$

where $\varepsilon_{r}^{\prime}$ and $\varepsilon_{r}^{\prime \prime}$ are the real and imaginary parts of the relative dielectric constant of the sample respectively and $C$ is a large constant. The variables $f$ and $Q$ are the resonance frequency and the quality factor respectively of the cavity after the sample has been introduced.

For the perturbation formulas in (1) to be accurate, the diameter of the cylinder of seawater must be less than $0.2 \mathrm{~mm}$. The constant $C$ appearing in (1) is related to the electromagnetic properties of the cavity without the seawater sample. This constant can be calculated directly from Maxwell's equations and then using the dimensions of the cavity, and the dimensions and dielectric constant of the slender tube. It can be shown that the constant $C$ in the perturbation limit is directly proportional to the volume of the sample. Thus if a measurement accurate to $1.0 \%$ is needed, the volume of the tube must be known to $1.0 \%$. A diagram of the cavity cross-section is shown in Figure 1.

\section{INITIAL MEASUREMENT SETUP}

A cylindrical cavity with a diameter of $16.214 \mathrm{~cm}$ and height of $7.142 \mathrm{~cm}$ was machined from brass. Borosilicate glass tubing with a $3 \mathrm{~mm}$ O.D. and a $0.1 \mathrm{~mm}$ I.D. was inserted down the axis of the cavity. Gaskets were placed at joints in anticipation of future immersion experiments to control the temperature. Two adjustable small microwave coupling loops were placed in the upper lid of the cavity. The cavity was connected to an HP8722D network analyzer (ANA) whose 


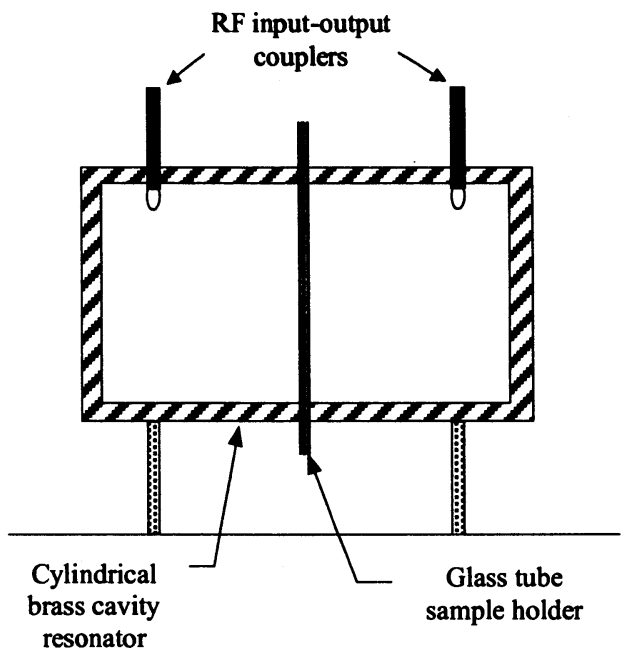

Figure 1. Microwave cavity.

frequency was stabilized by an Agilent 53132A ultra stable counter. This system provided the necessary clock frequency input to measure frequency shifts on the order of $70 \mathrm{kHz}$ accurately.

Because of the small ID of the glass tube, seawater would not readly enter the tube. Nitrogen gas under a pressure of about two atmospheres was used to force the seawater into the top of the tube. A vacuum pump was also used to suck the liquid out of the bottom of the tube. This arrangement was also useful for drying the tube after washing it with distilled water. The temperature of the seawater and the cavity were monitored with Barrett thermistors having an accuracy of $0.1^{\circ} \mathrm{C}$. The cavity was placed in a styrofoam container to stabilize the temperature during measurement. Using this type of setup, only seawater dielectric constants at room temperatures could be measured; repeating the same conditions was difficult. Results of these dielectric measurements appear in [4].

\section{Temperature ConTROlled SeTUP}

A box shaped container was constructed out of 0.5 inch thick Lexan to hold the cavity and the cooling liquid. A glass super-structure was designed to introduce the seawater under pressure into the slender tube in the cavity. All glass parts that were in prolonged contact with the seawater have been made of fused quartz. Although borosilicate glass tubes are easier to manufacture, they can introduce potassium ions into the seawater and change its dielectric constant. The cavity was kept at a constant temperature by filling the Lexan container with a mixture of ethylene gycol and distilled water so the mixture would not freeze at low temperatures. A Lauda Proline circulator was purchased to heat or cool the liquid as needed.

The Lauda unit specifies that it will keep the liquid to $0.01 \mathrm{C}$ over the desired temperature range of $0^{\circ} \mathrm{C}$ to $30^{\circ} \mathrm{C}$. Three US Sensor thermistors which are accurate to $0.01^{\circ} \mathrm{C}$ were purchased. Two were installed to measure the cavity temperature and one for the measurement of the seawater just before it enters the cavity. A Keithley Multimeter is being used to record the thermistor temperatures. Provision was made to pump the air out of the cavity chamber and replace it with dry

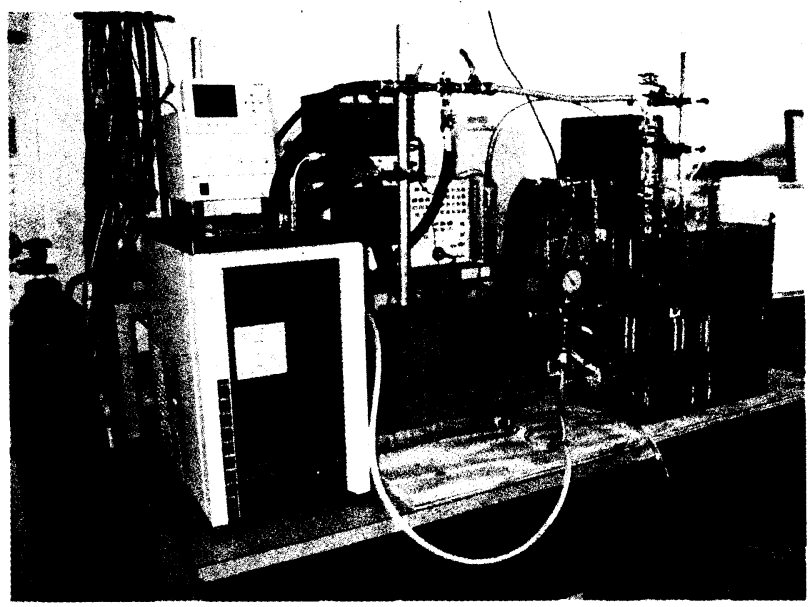

Figure 2. Seawater dielectric measurement setup.

nitrogen. Finally, a Visual Basic program has been developed to automatically collect the data from the ANA and the Keithley so that the measurements can be run over an extended time period. A photograph of the temperature controlled cavity setup is shown in Figure 2.

As was pointed out earlier, the volume of the seawater must be known as accurately as possible. One approach is to measure the area of the tube at each end. If it is assumed that the average of the two areas will provide an average area for the portion of the tube in the cavity, the volume can be computed by using the length of the tube in the cavity times this average area. The technique was implemented by photographing the tube ends with a Keyence Digital Microscope using 1000 times magnification. Using rulers in the image, the size of a pixel in the image was computed and the number of pixels in the tube opening was counted. The images of the top and bottom are shown in Figure 3. Another approach, in which the tube is filled with mercury and than the mercury in the tube is weighted, will be tried at a later time.

\section{CAVITY Stability MEASUREMENTS}

The planned procedure for making a series of measurements will be as follows: first, the Lauda circulator will be programmed to run through a series of temperatures;

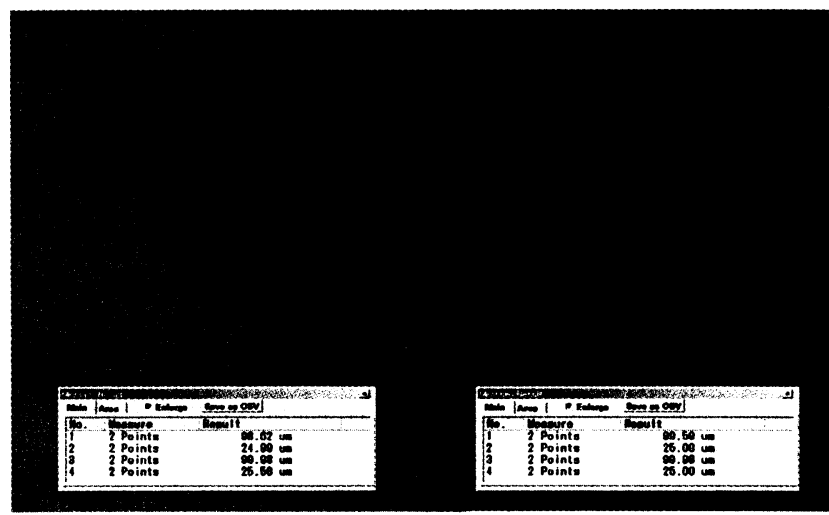

Figure 3. Glass sample tube top and bottom magnified pictures. 
second, at each temperature the cavity will be allowed to stabilize and then measurements of $f_{o}$ and $Q_{o}$, will be made. Finally, the seawater will be introduced into the cavity and measurements of $f$ and $Q$ at each temperature will be repeated. For this procedure to be successful, the cavity frequency and $Q$ must remain in a stable condition for the complete time the measurements are being made. Depending on the temperature

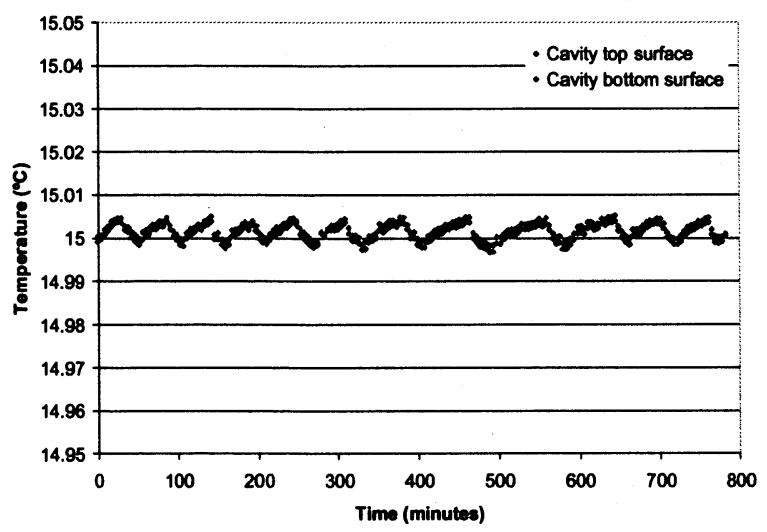

(a)



(b)

Figure 4. Cavity stability for (a) temperature and (b) frequency. increments and the time to stabilize at each new temperature, this time could be as long as 12 hours.

Stability measurements have been made over a 13 hour time period. The graphs of cavity temperature and cavity frequency are shown in Figures $4 \mathrm{a}$ and $4 \mathrm{~b}$ as a function of time. An examination of Figure 4a shows that over the 13 hour period the cavity temperature variation is less than $0.02^{\circ} \mathrm{C}$. From Figure $4 \mathrm{~b}$ it is seen that the cavity frequency varies by about $1 \mathrm{kHz}$ over a 13 hour period. Past measurements have shown that the presence of seawater shifts the cavity frequency by about $70 \mathrm{kHz}$. If the cavity frequency drifts by $700 \mathrm{~Hz}$ between the empty and filled tube condition, this would lead to a $1.0 \%$ error. Understanding how to further stabilize the frequency drift is now being investigated. In addition, stability measurements of the $\mathrm{Q}$ are also about $1.0 \%$.

\section{SUMMARY}

A temperature controlled microwave cavity has been constructed to measure the complex dielectric constant of seawater at a salinity ranging from 10 to $40 \mathrm{psu}$ and at a temperature between $0^{\circ} \mathrm{C}$ to $30^{\circ} \mathrm{C}$. Initial measurements of the system have shown that the resonant frequency and $\mathrm{Q}$ factor of the cavity can be kept at constant values to better than $1 \%$ of an extended time period (approximately 13 hours).

\section{REFERENCES}

[1] L.A. Klein and C.T. Swift, "An improved model for the dielectric constant of sea water at microwave frequencies," IEEE Trans. Antennas Propagat., vol. AP-25, no. 1, pp. 104-111, January 1977.

[2] W. Ho and W.F. Hall, "Measurements of the dielectric properties of seawater and $\mathrm{NaCl}$ solutions at $2.65 \mathrm{GHz}$," J. Geophys. Res., vol. 78, no. 27, pp. 6301-6315, September 1973.

[3] W. Ho, W. Love and M.J. van Melle, "Measurements of the dielectric properties of sea water at $1.43 \mathrm{GHz}$," NASA Contractor Report CR$2458,1974$.

[4] R. H. Lang, C. Utku and D. M. Le Vine, "Measurement of the dielectric constant of seawater at L-band," Proc. of the Inter. Geosci. Remote Sens. Symp. Vol 1, pp. 19-21, July 2003.

[5] S. Blanch and A. Aguasca, "Dielectric permittivity measurements of sea water," Proc. of the ESA Euro STARRS, WISE and LOSAC Campaign Workshop, ESA-SP25, 2003. 